\title{
Central synaptic mechanisms underlie short-term olfactory habituation in Drosophila larvae
}

\author{
Aoife Larkin, ${ }^{1,2}$ Somdatta Karak, ${ }^{3}$ Rashi Priya, ${ }^{4}$ Abhijit Das, ${ }^{3}$ Champakali Ayyub, ${ }^{3}$ \\ Kei Ito, ${ }^{5}$ Veronica Rodrigues, ${ }^{3,4}$ and Mani Ramaswami ${ }^{1,2,3,4,6}$ \\ ${ }^{1}$ School of Genetics and Microbiology, Smurfit Institute of Genetics and Trinity College Institute of Neuroscience, Trinity College \\ Dublin, Dublin 2, Ireland; ${ }^{2}$ School of Natural Sciences, Smurfit Institute of Genetics and Trinity College Institute of Neuroscience, Trinity \\ College Dublin, Dublin 2, Ireland; ${ }^{3}$ Department of Biological Sciences, TIFR, Mumbai 400005, India; ${ }^{4}$ National Centre for Biological \\ Sciences, TIFR Centre, Bangalore 560085, India; ${ }^{5}$ Institute of Molecular and Cellular Biosciences, The University of Tokyo, \\ Toyko 1130032, Japan
}

\begin{abstract}
Naive Drosophila larvae show vigorous chemotaxis toward many odorants including ethyl acetate (EA). Chemotaxis toward EA is substantially reduced after a 5-min pre-exposure to the odorant and recovers with a half-time of $\sim 20 \mathrm{~min}$. An analogous behavioral decrement can be induced without odorant-receptor activation through channelrhodopsin-based, direct photoexcitation of odorant sensory neurons (OSNs). The neural mechanism of short-term habituation (STH) requires the (1) rutabaga adenylate cyclase; (2) transmitter release from predominantly GABAergic local interneurons (LNs); (3) GABAA receptor function in projection neurons (PNs) that receive excitatory inputs from OSNs; and (4) NMDA-receptor function in PNs. These features of STH cannot be explained by simple sensory adaptation and, instead, point to plasticity of olfactory synapses in the antennal lobe as the underlying mechanism. Our observations suggest a model in which NMDAR-dependent depression of the OSN-PN synapse and/or NMDAR-dependent facilitation of inhibitory transmission from LNs to PNs contributes substantially to short-term habituation.
\end{abstract}

[Supplemental material is available online at http://www.learnmem.org.]

Experience-induced plasticity of synapses is believed to be a fundamental mechanism of learning and memory (Malenka and Bear 2004). However, central synaptic changes that underlie memory have not been clearly defined, even for relatively simple nonassociative learning processes such as habituation (Wilson and Linster 2008; Glanzman 2009).

During habituation, unreinforced exposure to a repeated or prolonged stimulus results in a reversible decrease in response to that stimulus (Thompson and Spencer 1966; Rankin et al. 2009). Habituation probably serves as an important building block for more complex cognitive function. By allowing unchanging or irrelevant stimuli to be ignored, it allows cognitive resources to be focused on more salient stimuli (Groves and Thompson 1970; Fabiani et al. 2006; Wilson and Linster 2008; Rankin et al. 2009).

The neural basis of short-term habituation (STH) is best studied in the marine snail, Aplysia californica. Here STH (lasting $\approx 30 \mathrm{~min}$ ) of the defensive gill-withdrawal reflex in response to tactile stimulation of the siphon is thought to arise from presynaptic depression of transmitter release at sensorimotor synapses (Bailey and Chen 1983, 1988; Armitage and Siegelbaum 1998). However, even here, presynaptic plasticity may not be cellautonomous, potentially requiring, for instance, activity of yetto-be-identified interneurons (Glanzman 2009).

Several forms of habituation have been described in Drosophila and are often shown to require the function of genes that regulate cAMP-dependent forms of associative memory

${ }^{6}$ Corresponding author.

E-mail mani.ramaswami@tcd.ie; fax 353-(1) 896-3183.

Article is online at http://www.learnmem.org/cgi/doi/10.1101/lm.1839010.
(Duerr and Quinn 1982; Engel and Wu 1996, 2009; Cho et al. 2004). For instance, habituation of proboscis extension reflex as well as odor-evoked startle reflex in adult Drosophila requires rutabaga (rut)-encoded $\mathrm{Ca}^{2+}$ /calmodulin-sensitive adenylyl cyclase (Livingstone et al. 1984; Levin et al. 1992; Asztalos et al. 2007; Engel and $\mathrm{Wu}$ 2009). In addition, habituation of the ethanolinduced startle response requires the shaggy/GSK-3 signaling pathway (Wolf et al. 2007). Despite such pioneering observations, the mechanisms of these various forms of habituation, even whether the primary neuronal changes are purely sensory or involve plasticity of central synapses (involving centrally located interneurons that may integrate various different kinds of modulatory, inhibitory, and excitatory inputs), remain poorly understood (Engel and Wu 2009).

Recent advances in understanding the circuitry that underlies Drosophila olfactory behavior, as well as the development of new tools to perturb identified neurons in vivo, has opened the opportunity for understanding mechanisms of olfactory habituation at the level of the underlying neural circuitry (Hildebrand and Shepherd 1997; Vosshall and Stocker 2007; Olsen and Wilson 2008; Masse et al. 2009).

In the larval olfactory system, 21 olfactory sensory neurons (OSNs), each expressing a single odorant receptor (together with the broadly expressed Or83b co-receptor), synapse, respectively, onto 21 cognate projection neurons (PNs) within 21 glomeruli in the larval antennal lobe (AL) (Stocker 2008). Local, predominantly GABAergic interneurons (LNs) synapse widely within the antennal lobe, interlinking different glomeruli (Ramaekers et al. 2005; Chou et al. 2010). Various neuromodulatory synapses also form on the larval antennal lobe and mushroom body (Schroll et al. 2006). Thus, odorant-stimulated signals in sensory neurons are processed in the antennal lobe, modulated by motivational or 
emotional states, and relayed through projection neurons to higher brain centers.

Previous work has shown that in Drosophila larvae, olfactory chemotaxis decreases after odorant pre-exposure (Rodrigues 1980; Cobb and Domain 2000; Wuttke and Tompkins 2000; Boyle and Cobb 2005). Here, we show that this behavioral habituation, alternatively referred to as "adaptation" by some previous investigators, arises from mechanisms of synaptic plasticity. We demonstrate that odorant receptor activation is not necessary for olfactory habituation; however, local interneuron activity and projection neuron signaling is necessary. Our observations suggest a model in which habituation occurs by a pathway in which NMDA receptors in projection neurons signal depression of OSN-PN synapses and/or facilitation of LN-PN synapses.

\section{Results}

\section{Habituation of the olfactory response in Drosophila larvae}

We standardized the method of Cobb and Domain (2000) to efficiently measure and characterize olfactory responses and habituation in Drosophila larvae (Fig. 1A; Materials and Methods). Wild-type (Oregon- $R$ ) larvae are attracted by many odorants, including benzaldehyde, 3-hexanol, and ethyl acetate (EA), in agreement with previous observations (Monte et al. 1989; Boyle and Cobb 2005). Because chemotactic responses to EA were the strongest among the odorants tested, we selected this chemical for use in subsequent experiments.

Naive larvae were robustly attracted to EA (Fig. 1B). However, a 5-min pre-exposure to $10^{-2}$ EA substantially decreased chemotactic responses measured at multiple EA concentrations $\left(10^{-1}\right.$, $\left.10^{-2}, 10^{-3}\right)$. Particularly robust and consistent behavioral decrements were observed at a testing concentration of $10^{-3}$, when larvae were pre-exposed for $5 \mathrm{~min}$ to a $10^{-2}$ dilution of EA (Fig. 1C). We performed a series of subsequent experiments to characterize the features and mechanisms of this behavioral decrement, which we define as "habituation." Unless otherwise stated, all habituation experiments described hereafter tested larval chemotaxis at $10^{-3} \mathrm{EA}$ after a 5 -min pre-exposure at $10^{-2} \mathrm{EA}$.

This olfactory habituation recovered spontaneously in $<1$ h. Chemotactic responses, which fell to $\approx 25 \%$ of control levels
A
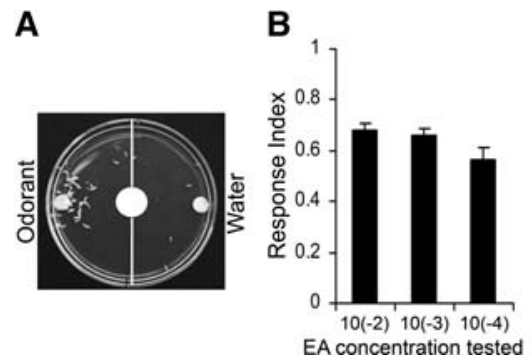

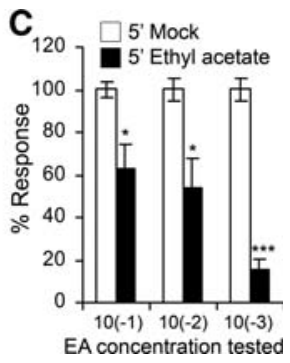

Figure 1. Inducing olfactory habituation in Drosophila larvae. $(A)$ Olfactory chemotaxis assays for larvae (larval plate test). Water was used as the solvent for ethyl acetate (EA). (B) Wild-type (Oregon- $R$ ) larvae were tested for olfactory responses to $10^{-2}, 10^{-3}$, and $10^{-4}$ concentrations of ethyl acetate (EA). Raw response indices (RI) are shown at each tested concentration: $\mathrm{RI}=[($ Number of larvae on odor side $)-($ number of larvae on water side)]/(Total number of larvae), for $10^{-2}$ EA $(n=7$ : each $n$ represents one batch of $20-60$ larvae), for $10^{-3}$ EA $(n=9)$, and for $10^{-4}$ EA $(n=6)$. (C) Olfactory response of mock (water)-exposed larvae. The white bar is higher than the olfactory response after 5-min exposure to $10^{-2}$ EA (black bars); responses were measured at $10^{-1}$, $10^{-2}$, or $10^{-3} \mathrm{EA}\left(^{*}\right) P<0.05 ;\left({ }^{* *}\right) P<0.001 ; n=6$ (repeats of experiments with 20-60 larvae per experiment). Data are expressed as mean \pm SEM.
5 min after pre-exposure, returned to near control levels after $\approx 40$ min of rest in the absence of odor (Fig. $2 \mathrm{~A}$ ).

To test if the initial decrement and subsequent recovery could be explained by motor fatigue or nonspecific odorant intoxication, we asked whether the olfactory response decrement observed was selective for the pre-exposed odorant. As shown in Figure 2B, larvae pre-exposed to EA showed relatively robust responses to propionic acid (PA) or butyl acetate (BA), odorants sensed by OSNs distinct from those that responded to EA (Fishilevich et al. 2005). This observation eliminates the possibility that reduced chemotaxis to EA observed after EA pre-exposure derives from larval motor fatigue or general toxicity.

To ask whether similar "short-term habituation" (STH) could be induced by direct depolarization of OSNs, we optically stimulated OSNs expressing the blue-light-activated cation channel channelrhodopsin (ChR2) (Schroll et al. 2006; Suh et al. 2007). The Or83b receptor subunit is widely used by almost all larval OSNs (Larsson et al. 2004; Ramaekers et al. 2005). Ten-minute photostimulation of Or83b-expressing OSNS led to reduced chemotaxis to multiple odorants such as EA and propyl alcohol (Fig. 2C, left panel). These light-induced response decrements recovered shortly after photostimulation ceased. In contrast to Or83b, the Or42a receptor is expressed in a specific subclass of OSN that mediates larval olfactory responses to isoamyl acetate but not to 2-propanol (Fishilevich et al. 2005). Ten-minute bluelight stimulation of Or42a>ChR2 larvae (expressing ChR2 in Or42a-positive OSNs) substantially reduced the subsequent olfactory response to isoamyl acetate, but not to 2-propanol (Fig. 2C, right panel). Together, these results indicate that behavioral decrements similar to those observed during habituation may be induced by action potential firing in OSNs without need for intracellular signaling pathways activated by odorant receptor activation.

One defining feature of behavioral habituation is its susceptibility to "dishabituation" by strong or noxious stimulation (Thompson and Spencer 1966; Engel and Wu 2009; Rankin et al. 2009). We asked whether olfactory STH showed this characteristic lability. A 1-min exposure to cold ( $4^{\circ} \mathrm{C}$ "cold shock") greatly improved the olfactory response of larvae habituated by a 5-minute pre-exposure to $10^{-2}$ EA (Fig. 2D). Cold shock also disrupted behavioral decrements observed after direct OSN depolarization (Fig. 2E). Direct depolarization of OSNs by $30^{\circ} \mathrm{C}$ exposure of Or $83 b>\operatorname{TrpA} 1$ larvae expressing the heat-activated TrpA1 cation channel caused a decrease in olfactory chemotaxis that was sensitive to brief cold exposure (Fig. 2E; Rosenzweig et al. 2005; Pulver et al. 2009). Importantly, a similar cold shock applied to naive larvae had no effect on their chemotaxis toward $10^{-3} \mathrm{EA}$. This indicates that cold shock causes loss of habituation, rather than general sensitization to olfactory stimuli (Fig. 2F).

Together these behavioral observations indicate that larval olfactory STH has commonalities with other forms of behavioral habituation (Engel and Wu 2009). The phenomenon of dishabituation may additionally constrain the range of potential neurophysiological mechanisms for habituation. For instance, a hypothetical activity-dependent run-down of voltage-gated sodium channels in sensory neurons could conceivably account for STH, but is unlikely to be rapidly reversed by a brief cold shock.

\section{Local interneurons are required for larval olfactory STH}

A simple possible explanation for STH is that it arises because projection neurons respond less efficiently to sensory neuron input. This could arise from homosynaptic depression of the OSN-PN synapse and/or alternative circuit mechanisms such as increased local inhibition. In adult Drosophila, cholinergic OSN-PN synaptic transmission is modulated by inhibition from local GABAergic 

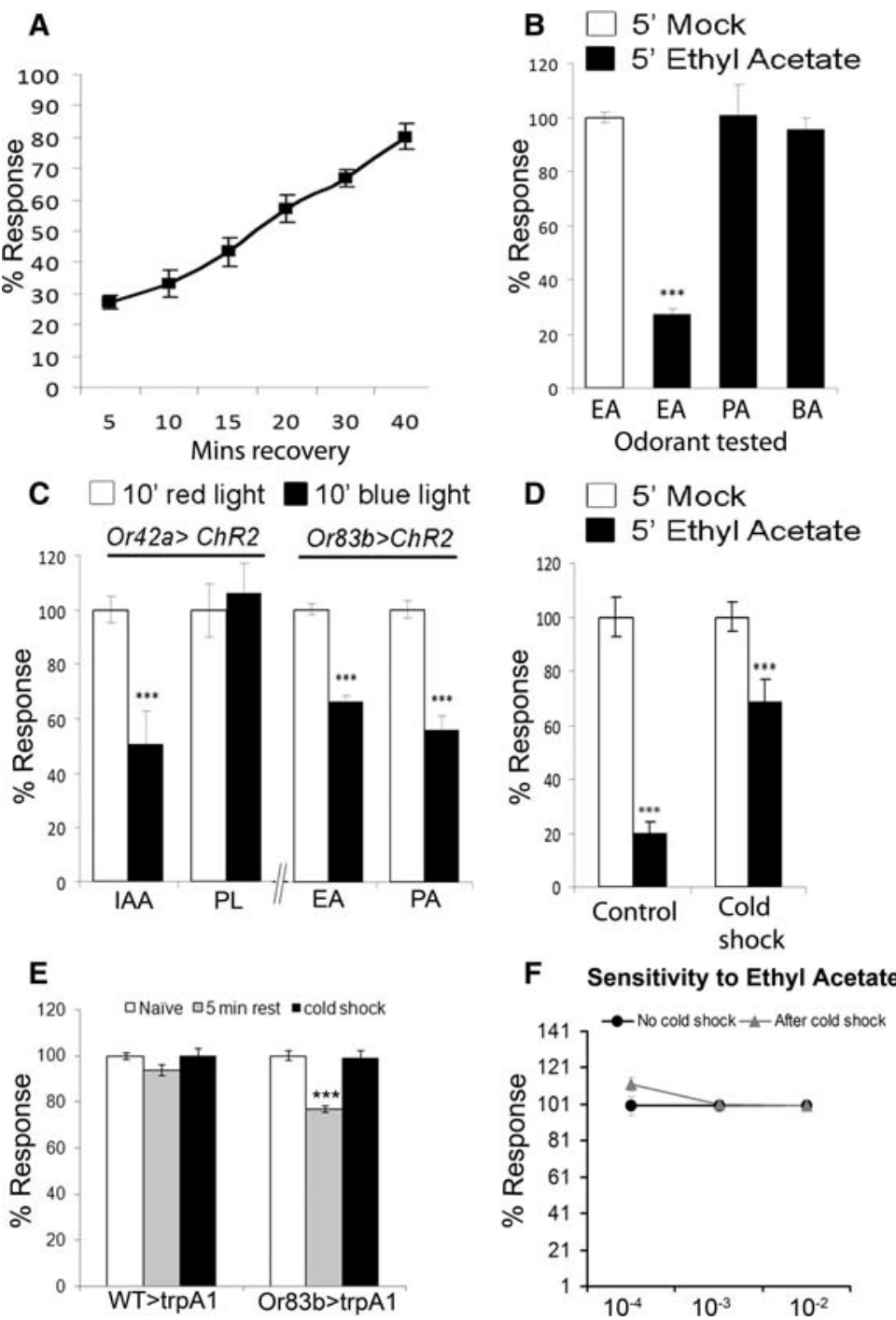

Figure 2. Properties of olfactory habituation in Drosophila larvae. (A) Habituation recovers in $<1$ h. Responses of wild-type (Canton-S) larvae to $10^{-3}$ ethyl acetate (EA) at different time points after 5 -min exposure to either $10^{-2}$ EA or water (mock exposed). Response indices were calculated as a percentage of the average mock-exposed response (percent response) at each time point. (B) Olfactory habituation shows odor selectivity. Canton-S (wild-type) larvae were exposed to a concentration of $10^{-2} \mathrm{EA}$ (or water) for $5 \mathrm{~min}$ and immediately tested for olfactory responses to $10^{-3} \mathrm{EA}, 10^{-3}$ propionic acid (PA), or $10^{-3}$ butyl acetate (BA); $n=10$. (C) Forced depolarization of odorant sensory neurons is sufficient to induce olfactory habituation. (C, left panel) Blue-light-driven global photoactivation ORNs (in Or83bGal4 > UAS ChR2 larvae) reduces larval olfactory response to multiple odorants such as ethyl acetate (EA) and propionic acid (PA) when compared to red-light-exposed controls. This behavioral decrement recovers over a short time period. (C, right panel) Blue-light stimulation of channelrhodopsin in the OR42a expressing ORN (in Or42aGal4> UAS ChR2 larvae) selectively reduces subsequent response to isoamyl acetate $\left(\mathrm{IAA}, 10^{-3}\right)$ but not 2-propanol $\left(\mathrm{PL}, 10^{-3}\right)$ when compared with unexposed controls. (D) A 1-min cold shock disrupts habituation. Wild-type (Oregon-R) larvae were exposed to $10^{-2} \mathrm{EA}$ (or water) for $5 \mathrm{~min}$, then tested for responses to $10^{-3} \mathrm{EA}$ after $1 \mathrm{~min}$ in either lukewarm ("warm wash") or $4^{\circ} \mathrm{C}$ ("cold wash") water; $n=6$. Data are expressed as mean \pm SEM. (***) $P<$ 0.001 . Each $n$ represents one experiment with between 20 and 60 larvae. (E) Forced depolarization using the heat-activated TrpA1 channel expressed in Or83b-positive OSNs also causes a small but significant reduction of the chemotactic response, which is also reversed by cold shock $(n=11$ sets for each bar). $(F)$ In naive animals not previously exposed to EA, cold shock does not alter olfactory chemotaxis to $10^{-3}$ dilution of EA ( $n=10$ sets for each bar).

interneurons (LNs). Because local inhibitory interneurons have also been observed in larvae, we investigated the nature of these neurons as well as their potential roles in the formation or expression of habituation memory.
In the adult antennal lobe, the enhancer-trap lines Gal4:LN1 and Gal4:LN2 (Sachse et al. 2007; Okada et al. 2009; Tanaka et al. 2009) label subsets of GABAergic local interneurons. We found that these also mark larval olfactory interneurons (Fig. 3A-E). MARCM methods (mosaic analysis with a repressible cell marker) allowed us to visualize single LNs and find that these innervate multiple glomeruli (Fig. 3E). In contrast, GH146-labeled PNs arborize in single glomeruli in the antennal lobe and connect to the higher centers in the brain (Fig. 3F). The two Gal4 lines identify different subsets of larval LNs: Gal4:LN1 marks five local interneurons in the larval antennal lobe, all of which are GABAergic (Fig. 3A,C). The Gal4:LN2 line marks about 12 local interneurons around the antennal lobe, and of these seven can be labeled by anti-GABA antibodies (Fig. 3B,D).

We asked whether perturbation of activity in the $L N 1$ or $L N 2$ class of local interneuron would affect olfactory habituation. The expression of active tetanus-toxin (TNTG) in LN1 or LN2 cells is predicted to constitutively reduce transmitter release from these neurons, while inactive control (TNTVIF) toxin should not (Sweeney et al. 1995). To ask whether reduced transmitter release from these neurons could affect shortterm habituation, we expressed either TNTG or TNTVIF in local interneurons and assessed STH in the resulting larval progeny.

We first examined consequences of expressing tetanus toxin in all LN1- and LN2-labeled cells. Control experiments showed that LN1, LN2>TNTVIF larvae expressing inactive tetanus toxin behaved and habituated like wild-type animals. In contrast, experimental LN1, $L N 2>T N T G$ larvae showed no detectable habituation following pre-exposure to ethylacetate (Fig. 4A). The effect was selective for the process of habituation, because $L N 1, L N 2>T N T G$ larvae showed near-normal, naive chemotactic responses at different concentrations of ethyl acetate (Fig. 4).

Expression of tetanus toxin in only the LN1 subset of interneurons was sufficient to reduce STH to ethyl acetate (EA). Thus, LN1>TNTG larvae showed strongly reduced STH when compared to LN1>TNTVIF controls. In contrast, $L N 2>T N T G$ and $L N 2>$ TNTVIF larvae showed normal STH (Fig. 4C). Given that the LN2 promoter appears to be considerably stronger than $L N 1$ in larval local interneurons (Fig. 4D), this selective effect of perturbing $L N 1$ indicates functional specialization among larval interneurons (Okada et al. 2009; Tanaka et al. 2009). 


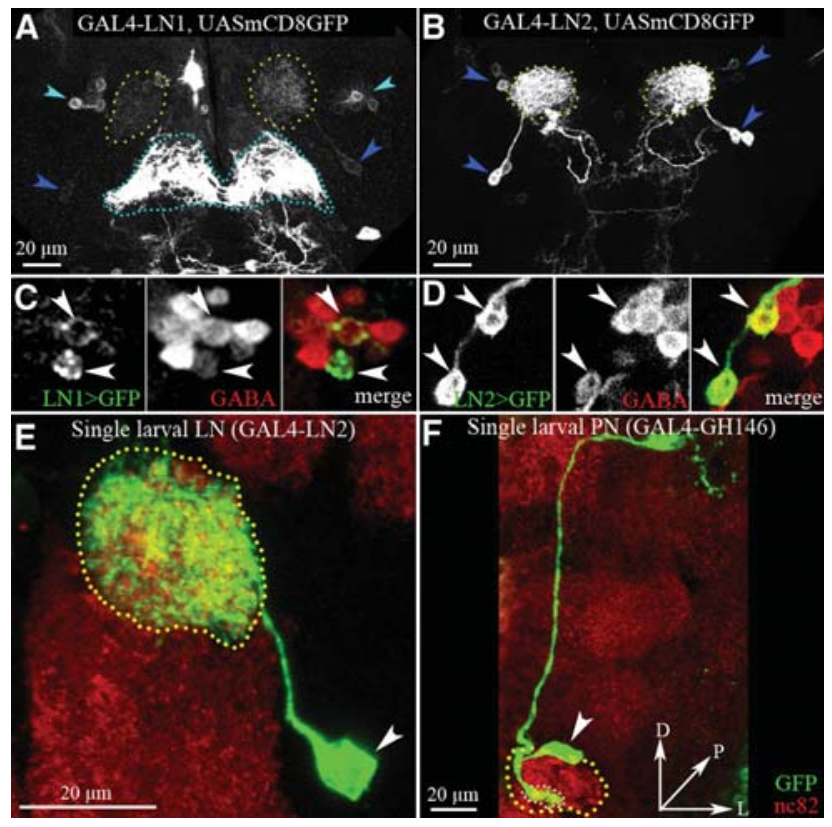

Figure 3. Neurons marked by $L N 1 \mathrm{Gal} 4$ and the $L N 2$ Gal4 lines in the larval antennal lobe. $(A, B)$ Anti-GFP labeling of CD8-GFP expressed in the larval brain under control of Gal4:LN1 $(A)$ or Gal4:LN2 (B). Cell bodies are indicated by arrowheads (cyan arrows show soma not contributing to the antennal lobe). The antennal lobes are outlined with a yellow dotted line, and the SOG (subesophageal ganglion) is outlined by a cyan dotted line. $(C, D)$ Larval brains double-immunolabeled against GABA (red) and GFP (green) in animals expressing membrane-targeted GFP under control of Gal4:LN1 (C) or Gal4:LN2 (D). White arrowheads indicate cells marked by the Gal4 line that are positive for GABA. (E) Example of single local interneuron labeled using the MARCM technique showing its multiglomerular projections into the larval antennal lobe (dashed outline). ( $F$ ) Example of single GH146 Gal4 marked larval projection neuron with a glomerulus-specific innervation of the antennal lobe and axonal projection higher brain centers. The antennal lobe is circled with a yellow dotted line, the glomerulus with a white dotted line.

To address the possibility that STH defects observed in LN1>TNTG larvae could arise from developmental defects, instead of functional deficits in neurotransmitter release, we used targeted expression of a dominant temperature-sensitive dynamin (UAS-Shibire ${ }^{t s}$ ) to acutely perturb transmitter release in the $L N 1$ neurons. Because dynamin is required for synaptic-vesicle recycling, cells expressing temperature-sensitive dynamins show a use-dependent depletion of synaptic vesicles and reduced transmitter release at or above restrictive temperatures (Kitamoto 2001, 2002). We used this technique to conditionally inhibit synaptic output from LN1-Gal4 neurons during odor exposure ("train"), consolidation ("rest"), or post-exposure olfactory testing ("test") and assessed the effect of these perturbations on induction or expression of habituation memory (Fig. 4E,F).

Control UAS-shi ${ }^{\text {ts1 }}$ larvae showed robust STH when trained or tested at temperatures restrictive for $s h i^{\text {ts1 }}$ function $\left(34^{\circ} \mathrm{C}\right)$ (Fig. 4E). Experimental LN1>Shi(ts1) larvae showed normal STH at permissive temperatures $\left(22^{\circ} \mathrm{C}\right)$ (Fig. $\left.4 \mathrm{~F}\right)$. However, LN1> Shi(ts1) larvae pre-exposed to $10^{-2} \mathrm{EA}$ at $22^{\circ} \mathrm{C}$ showed greatly reduced habituation if tested at restrictive temperatures $\left(34^{\circ} \mathrm{C}\right)$ (Fig. 4E). This shows that transmitter release from local interneurons is required for the display of reduced olfactory chemotaxis, characteristic of the habituated state.

While transmitter release from $L N 1$ neurons did not seem to be necessary during the period of "rest"-between training and testing, we found that transmitter release from LN1 neurons was also necessary during the pre-exposure/training period (Fig. 4E). Thus, when $L N 1>\operatorname{Shi}(t s 1)$ flies were pre-exposed to $10^{-2} \mathrm{EA}$ at $34^{\circ} \mathrm{C}$, they showed greatly reduced habituation (near naive olfactory chemotaxis) when tested at permissive temperatures. This unexpected requirement for $L N 1$ output during odor exposure indicates that STH occurs through a mechanism that is not restricted to OSNs.

\section{Larval olfactory STH requires GABA receptors on projection neurons}

Immunohistochemical data (Fig. 3) indicate that all larval LN1 neurons are GABAergic. If STH requires GABA release from LNs, then one would predict that it would require GABA receptors in postsynaptic neurons. We tested this prediction by asking whether knockdown of GABA receptors on particular sets of cells would affect habituation. We used the GH146-Gal4 line, which expresses in 16-18 out of the expected 21 larval PNs (Ramaekers et al. 2005; Stocker 2008) to drive an Rdl RNAi line (UAS-Rdli8$10 G)$, which has been shown to knock down Rdl-encoded $\mathrm{GABA}_{\mathrm{A}}$ receptors in the Drosophila nervous system (Liu et al. 2007, 2009). Knockdown of $R d l$ GABA $_{\mathrm{A}}$ Rs in GH146-marked PNs eliminates STH (Fig. 5A), without affecting mock-exposed larval olfactory responses to EA. These observations indicate that loss of STH observed following LN perturbations probably derives from defective GABAergic transmission in the antennal lobe.

\section{Larval olfactory STH requires NMDA receptors on projection neurons}

To further test whether synaptic changes in the antennal lobe were required for STH, we asked whether STH required the function of NMDA receptors, which have been previously shown to play a role in adult associative memory (Xia et al. 2005; Wu et al. 2007). Two Drosophila NMDAR homologs, $d N R 1$ and $d N R 2$, dimerize to form functional NMDARs with properties similar to those in vertebrates (Xia et al. 2005). We drove a validated transgenic RNAi construct (UAS-dsNR2) (Xia et al. 2005; Wu et al. 2007) in GH146-Gal4 expressing projection neurons and analyzed the effect on olfactory behavior. Knockdown of NMDARs on PNs eliminated larval STH to EA (Fig. 5B) without affecting mockexposed larval olfactory responses to EA.

Pointing to a requirement for central NMDARs, this experiment most clearly indicates that central synaptic mechanisms are necessary for short-term olfactory habituation.

\section{rutabaga is required for larval olfactory short-term habituation}

Previous work has shown that Drosophila rutabaga (rut) mutants defective in a calcium-calmodulin-dependent adenylate cyclase, an enzyme that detects the coincidence of calcium and G-protein signaling, show defects in a number of types of habituation and memory (Bourne and Nicoll 1993; Blum et al. 2009; Engel and Wu 2009; Gervasi et al. 2010). We asked if STH of olfactory chemotaxis was similarly dependent on rutabaga. We found that strongly hypomorphic, rut ${ }^{2080}$ mutant larvae show significantly reduced habituation in comparison to Oregon- $R$ larvae (Fig. 6A). Although the naive responses of rut ${ }^{2080}$ to EA appeared to be higher at $10^{-1}$ and $10^{-2}$ concentrations of EA, the responses of rut and control Oregon-R larvae were similar at the behaviorally relevant testing concentration $10^{-3}$ (Fig. 6B). Thus, rut appears to be required for habituation of the olfactory chemotactic response. This conclusion is strengthened by the observation that "leaky" expression of the rut ${ }^{+}$transgene in rut ${ }^{2080} ;$ UAS-rut $^{+}$larvae rescues the $r u t^{2080}$ defect in habituation. 
A
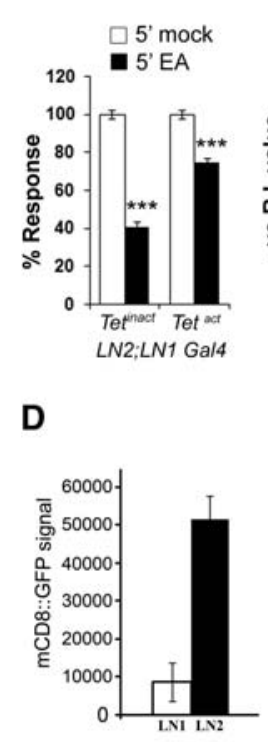

E LN1>shi(ts1)
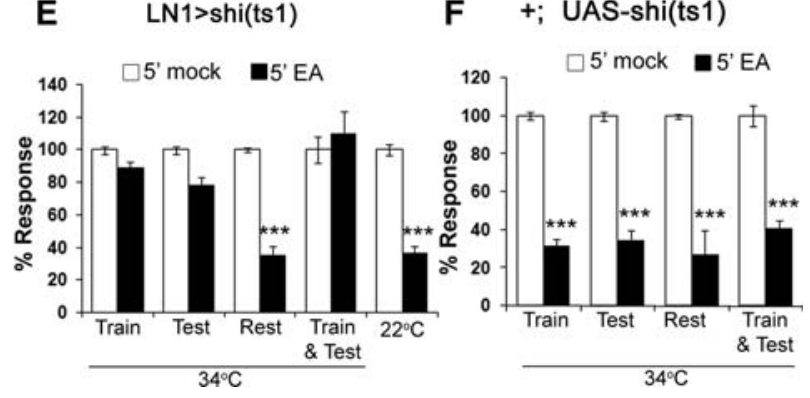

Figure 4. Transmitter release from $L N 1$ local interneurons is necessary for habituation. ( $A$ ) Responses of mock-exposed (white bars) and $10^{-2}$ EA-exposed (black bars) larvae expressing active (Tet act $_{\text {TNNT) }}$

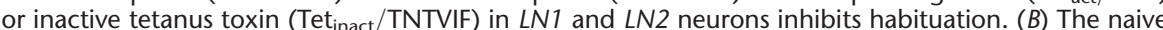
olfactory chemotactic response in LN1, LN2>TNT (black bars) not significantly different from LN1, LN2>TNT-VIF (white bars) larvae expressing inactive tetanus toxin in LN1 and LN2 interneurons. Thus, tetanus toxin expression in LNs blocks STH without altering the basal response to ethyl acetate at concentrations between $10^{-6}$ and $10^{-3}$. (C) Tetanus toxin expression in LN1-Gal4-positive neurons, but not in LN2-Gal4, inhibits short-term habituation. White bars show responses of mockexposed controls; black bars in larvae after pre-exposure to ethyl acetate (EA). (D) Gal4-LN2 drives reporter gene expression more efficiently than Gal4-LN1. GFP fluorescence in LN1>GFP and $L N 2>$ GFP antennal lobes quantified from confocal images taken under identical image acquisition settings. (E) In $L N 1>$ shi $^{\text {ts }}$ larvae pre-exposed to ethyl acetate, habituation memory is apparent if the animals are tested at $22^{\circ} \mathrm{C}$ (white bar is larger than the black bar). But STH is not displayed (white and black bars of similar size) if tested (test) at $34^{\circ} \mathrm{C}$, when output of $L N 1$ neurons is inhibited. Habituation is normal if $L N 1$ output is inhibited during "rest," the 5-min interval between pre-exposure (train) and testing. However, habituation is also invisible if output from LN1 is inhibited during training. Chemotactic responses of mock-exposed (white bar) and EA-exposed black bars are similar if $L N 1>$ shi $^{\text {ts } 1}$ larvae are trained at $34^{\circ} \mathrm{C}$, conditions restrictive for transmitter release from $L N 1$ neurons. (F) The UAS-Shit transgene does not alter expression of habituation memory in a wild-type (Canton-S) background. Data are expressed as mean \pm SEM; $n=10$ for all experiments, $\left({ }^{* * *}\right) P<0.001$.

However, phenotypic rescue observed with the UAS rut ${ }^{+}$ transgene in the absence of Gal4 expression prevented us from performing subsequent experiments to identify the cell type in which rut functions for larval $\mathrm{STH}$, as previously done for various forms of associative memory (Zars et al. 2000; Blum et al. 2009; Liu et al. 2009).

\section{Discussion}

\section{Properties of short-term olfactory habituation in the Drosophila larva}

Previous studies of larval habituation have not clearly discriminated between peripheral and central mechanisms. Indeed, the term "adaptation," better applied to sensory neuron changes such as receptor desensitization, has often been used interchangeably with the term "habituation," which is usually restricted to behavioral changes arising from central synaptic mechanisms (Cobb and Domain 2000; Wuttke and Tompkins 2000; Boyle and Cobb 2005; Linster et al. 2007; Wilson and Linster 2008).

The form of larval olfactory STH characterized here displays at least some of the defining behavioral characteristics of habituation (Thompson and Spencer 1966; Engel and Wu 2009; Rankin et al. 2009). First, there is a behavioral decrement in response to repeated or sustained application of a particular stimulus (Fig. 1). Second, STH shows spontaneous recovery with time in the absence of the habituating stimulus (Fig. 2A). And third, STH is susceptible to dishabituation when habituated larvae are presented with of a strong or noxious stimulus (Fig. 2D). The property of dishabituation is particularly significant, as an important way of distinguishing between habituation and either fatigue or sensory adaptation. Dishabituation shows that the habituated animal retains the capability to respond and suggests that the attenuated behavioral response arises from some form of active suppression. Thus, the behavioral data suggest (1) that the term "habituation" may be better used in place of "adaptation," while referring to the behavioral phenomenon that we study here; and (2) that STH probably arises from central synaptic mechanisms, rather than sensory neuron adaptation.

\section{Central mechanisms underlie larval olfactory short-term habituation}

Three main lines of data support our conclusion that STH arises from a central synaptic mechanism that resides in the antennal lobe, rather than from adaptation of olfactory receptor signaling in the OSN. First, behavioral decrements similar to STH can be induced by direct depolarization of OSNs, indicating that STH may potentially be induced by processes stimulated by activation actionpotential firing in OSNs, independently of olfactory receptor activation (Fig. 2C). Second, and more striking, STH requires synaptic-vesicle exocytosis from local interneurons during the process of odorant exposure, when STH is being established (Fig. 4E). This requirement is incompatible with an exclusively sensory mechanism. Third, STH requires the function of NMDA receptors on postsynaptic projection neurons. This last observation also provides a particularly strong argument for a synaptic mechanism, indicating a need for plasticity of OSN and/or LN synapses made onto dendrites of projection neurons in the antennal lobe. Given that OSNs are excitatory and LNs are primarily inhibitory, it appears most likely that NMDAR functions in PNs to depress excitatory OSN-PN synapses and/or to potentiate inhibition by strengthening the LN-PN synapse. We suggest that the LN-PN mechanism may be involved because (1) LN transmission seems necessary for both induction and expression of habituation (Fig. 4); and (2) the process of dishabituation could be attractively explained as arising from the inhibition of local inhibitory synapses through descending neuromodulation. A requirement for facilitation of the LN-PN synapse would be consistent with previous studies showing that adult-long-term olfactory habituation is associated with an increase in odor-evoked calcium fluxes in GABAergic processes within the Drosophila antennal lobe (Sachse et al. 2007). 

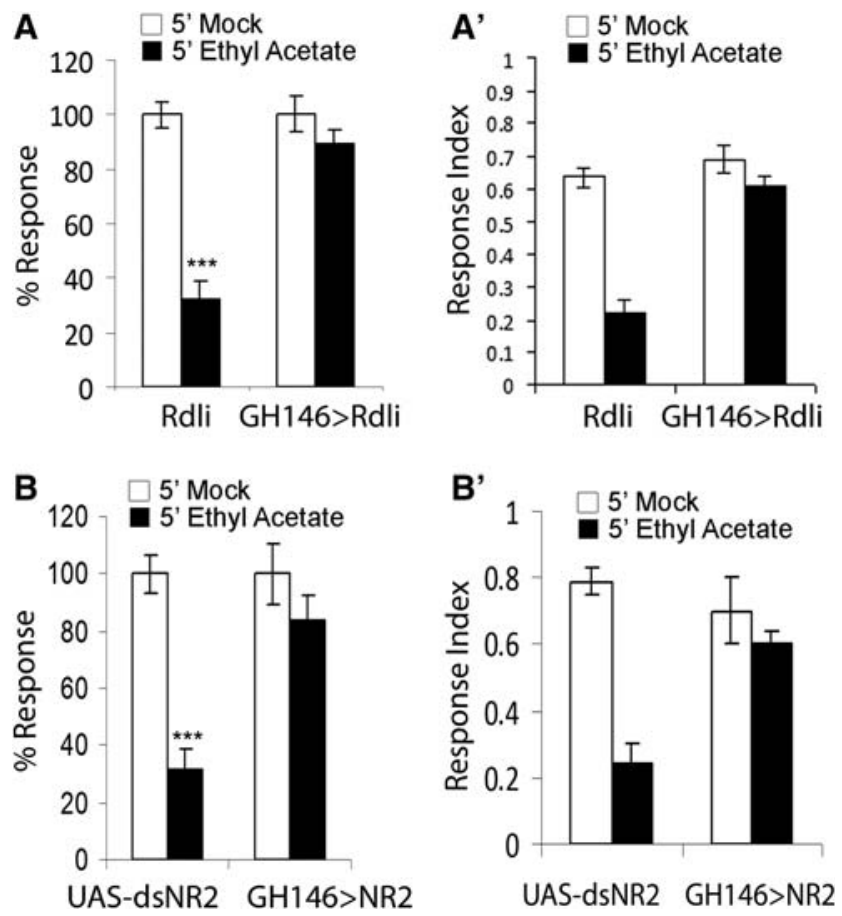

Figure 5. Effects of knockdown of receptors on PNs. Larvae were exposed to $10^{-2}$ ethyl acetate (EA; or water as a control) for $5 \mathrm{~min}$ and then tested for olfactory responses to $10^{-3}$ EA. Response indices are shown in $A^{\prime}$ and $B^{\prime}$, or were calculated as a percentage of the average control/mock-exposed response (percent response in $A$ and $B$ ). ( $A$ ) Silencing $\mathrm{GABA}_{\mathrm{A}} \mathrm{R}$ function in PNs (GH146-Gal4/UAS-Rdli8-10G) abolished larval olfactory STH $\left[\left(^{* * *}\right) P<0.001\right]$ without $\left(A^{\prime}\right)$ affecting olfactory responses. (B) Silencing NMDAR function in PNs (GH146-Gal4) $U A S-d s N R 2$ ) abolished larval olfactory STH without ( $\left.B^{\prime}\right)$ affecting olfactory responses. Data are expressed as mean \pm SEM.

Based both on experimental and theoretical arguments, we suggest a simple model for short-term olfactory habituation. We stress that this is a model, and being based on a preliminary analysis, we do not claim to have ruled out additional major contributing mechanisms.
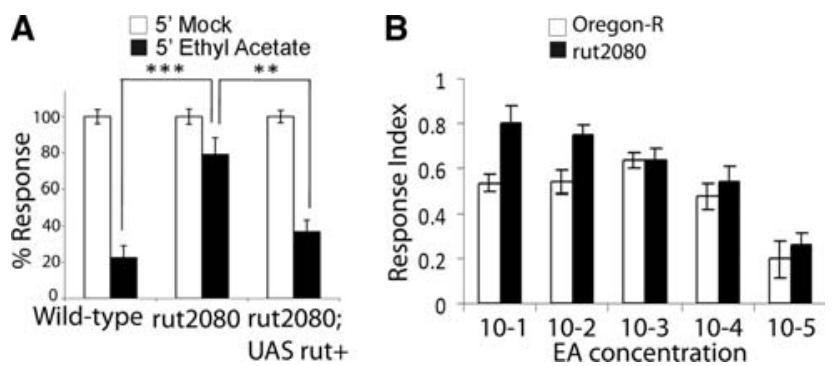

Figure 6. rut is required for larval olfactory STH. (A) Larvae (homozygous rut2080, Oregon- $R$, or rut2080; UAS-rut/UAS-rut) were exposed to $10^{-2}$ ethyl acetate (EA; or water as a control) for 5 min and then tested for olfactory responses to $10^{-3}$ EA $5 \mathrm{~min}$ after exposure. Response indices were calculated as described in the text. Significant differences are seen between rut and Oregon- $\mathrm{R}$ control flies using the Student's $t$-test $\left(n=8-12 ;{ }^{* * *} p<0.001\right)$. Leaky expression of rut in rut 2080 ; UAS-rut/UAS-rut larvae significantly improved STH in rut2080 (**P= $0.0081)$. (B) Larval (rut2080 or Oregon- $R$ ) olfactory responses to varying concentrations of EA $\left(10^{-1}\right.$ to $\left.10^{-5}\right)$. Raw response indices are shown $(n=5-10)$. Data are expressed as mean \pm SEM. Each $n$ represents one experiment with between 20 and 60 larvae.
We suggest that during initial odorant pre-exposure, dendritic NMDA receptors on projection neurons detect and respond to membrane depolarization occurs coincident with transmitter release from LNs. Calcium entry through dendritic NMDA receptors may trigger a local retrograde signal required for facilitation of transmitter release from the LNs. Although our existing data do not rule out functions for rutabaga in higher larval brain centers, we suggest that either the generation of a retrograde signal in PN dendrites or the presynaptic response of LNs to this signal could be dependent on the rut adenylate cyclase. In habituated animals, facilitation of GABA release would reduce odor-evoked projection neuron outputs to higher brain centers, thereby reducing olfactory behavior. As NMDAR signaling would only occur at active glomeruli, this mechanism can account not only for the observed odor selectivity of habituation (Fig. 2), but also the instances of cross-habituation that have been previously described (Boyle and Cobb 2005).

Such a model also naturally suggests a hypothesis for the mechanism of dishabituation: namely, that dishabituating stimuli cause release of neuromodulators that act to reduce GABA release from local inhibitory synapses.

\section{Relevance to other forms of habituation}

Given the remarkable similarities in the anatomical organization of insect and mammalian olfactory systems (Vosshall and Stocker 2007), a significant conservation of olfactory mechanisms would be expected. In rodents, at least two forms of habituation have been described, lasting 2-3 and 30-60 min, respectively: the latter equivalent in timescale to larval STH that we describe (McNamara et al. 2008; Wilson and Linster 2008). Consistent with a similar underlying mechanism, the more persistent form of olfactory habituation can be blocked by an N-methyl-Daspartate (NMDA) receptor antagonist in the olfactory bulb, a structure homologous to the insect antennal lobe (McNamara et al. 2008). Thus, larval STH that we describe has some similarities to a previously characterized form of mammalian olfactory habituation. Analysis of the underlying mechanisms is therefore likely to provide directly transferable insights in mammalian olfaction. Our data make the prediction that the activity of mammalian olfactory interneurons, either periglomerular or granule cells, is critical for the establishment and display of at least one timescale of olfactory habituation.

In addition to providing some insight into mechanisms of olfactory habituation in mammals, it possible that circuit mechanisms of larval olfactory habituation are relevant to other forms of behavioral habituation. In at least three previous instances, increased inhibition has been associated with attenuated behavior. For example, habituation of an escape reflex mediated by the lateral giant fibers in the crayfish has been associated with enhanced GABAergic transmission onto giant fibers (Krasne and Teshiba 1995). Similarly, LTP of inhibitory synapses controlling excitability of the Mauthner cell has been associated with reduced escape behavior in goldfish (Oda et al. 1998). Furthermore, ethanol, a potentiator of GABA synapses, has been shown to enhance habituation of a motor pathway in the frog spinal cord (Glanzman and Epperlein 1981). Could these different instances of habituation all involve circuit mechanisms similar to those used in Drosophila larval olfactory behavior?

In all brain regions, principal/projection neurons are subject to inhibitory feedback modulation and a pathway that has been appreciated as potentially essential for neuronal homeostasis (Kullmann and Lamsa 2007; Lisman et al. 2008). Potentiation of inhibitory feedback triggered by the pattern of principle cell activation would be predicted to preferentially dampen this particular output pattern. Thus, the circuit mechanism we suggest is 
theoretically generalizable to other and more complex forms of habituation. Further experiments will be required to determine the validity of this very testable hypothesis.

The importance of habituation has been underlined by the fact that deficits in sensory gating and pre-pulse inhibition (PPI), processes with similarities to habituation, have been linked with various neurological problems, including autism (Ornitz et al. 1993) and schizophrenia (Freedman et al. 1991; Ludewig et al. 2003; Meincke et al. 2004). Indeed, a circuit model for understanding schizophrenia has specifically proposed that altered negative feedback in the hippocampus may underlie both positive and negative symptoms of schizophrenia (Lisman et al. 2008).

In addition, defects in habituation or habituation-like processes have been described in Fragile X syndrome (Frankland et al. 2004) and migraines (Siniatchkin et al. 2003). It has also been shown to have important effects relating to learning disabilities (Slaats-Willemse et al. 2003), age-related changes in learning (Fabiani et al. 2006), and substance abuse (Hunt and Morasch 2004). If mechanisms of olfactory habituation prove to be general, then studies of olfactory plasticity may prove relevant for other forms of cognition as well as for human neurological disease.

\section{Materials and Methods}

\section{Drosophila stocks}

Fly stocks are grown on standard medium containing yeast. Flies were grown at $25^{\circ} \mathrm{C}$ unless mentioned otherwise. The Canton-S (CS) strain was the wild type in all the experiments. (See list of fly strains in Table 1.)

\section{Measuring larval olfactory responses}

The olfactory response tests were based on Monte et al. (1989), which was, in turn, based on that of Rodrigues (1980) and Aceves-Pina and Quinn (1979). Two small plastic caps (to exclude direct contact of odorants with larvae) were placed on opposite sides of a $9-\mathrm{cm}$ Petri dish covered with $\approx 10 \mathrm{~mL}$ of $2 \%$ agar. Each plastic cap contained a 5-mm filter paper disk with $25 \mu \mathrm{L}$ of either distilled water (control) or odor, to create an odor gradient. Control and odor sides were rotated from dish to dish. Serial dilutions of odorants resulted in concentrations of $10^{-1}(1: 10), 10^{-2}$ (1:100), $10^{-3}(1: 1000)$, and so on. Immediately after odorant placement, third-instar feeding stage larvae (between $\approx 20$ and 55 ) were placed in the center (within a 2 -mm-diameter circle) of the dish. The lid of the Petri dish was replaced, and larvae were allowed to wander for $5 \mathrm{~min}$, after which the numbers of larvae on each side of the dish (either odor or control) were counted.

Table 1. List of fly strains

\begin{tabular}{ll}
\hline Fly strain & \multicolumn{1}{c}{ Source } \\
\hline UAS-TNTG & Sean Sweeney, Cambridge, UK \\
UAS-TNTVIF & Sean Sweeney, Cambridge, UK \\
UAS-shits & Toshi Kitamoto, University of lowa \\
UAS-ChR2 & Andre Fiala, University of Goettingen \\
Rdli8-10G (UAS-Rdl-RNAi) & Ron Davis, Baylor College \\
UAS-dsNR2 (RNAi line against & Tim Tully, DART Neurosciences \\
NR2) & Bloomington Stock Center (\#6926) \\
UAS-syt-GFP & Leslie Vosshall, Rockefeller University \\
OR83b-Gal4 & Leslie Vosshall, Rockefeller University \\
Or42a-Gal4 & Reinhard Stocker, University of \\
GH146-Gal4 & Fribourg \\
rut ${ }^{2080}$ & Martin Heisenberg, University of \\
rut ${ }^{2080 ;}$; UAS rut & Wuerzburg \\
UAS TrpA1 & Martin Heisenberg, University of \\
& Wuerzburg \\
\hline
\end{tabular}

Any larvae that remained in the circular area marked out in the center of the plate were not counted.

Response indices (RI) were calculated as follows: ( $n_{\text {odor half }}-$ $\left.n_{\text {control half }}\right) / n_{\text {total }}$. In this way, an RI between -1 and 1 is obtained, where 1 represents total attraction (all larvae were on the side with the odor) and -1 represents total repulsion (all larvae were on the side with the control). Any graphs where percentages (\% Response) are shown represent responses expressed as a percentage of the control mock (water)-exposed response. All behavioral experiments were conducted in a temperature- and humiditycontrolled room $\left(25^{\circ} \mathrm{C}, 50 \%\right.$ humidity) under red light. Behavioral experiments for Rdli and NMDAR were done and performed double-blinded. Data are represented by the mean and standard error of the mean $( \pm$ SEM) from at least 10 replicates.

\section{Inducing short-term olfactory habituation in the larva}

This olfactory habituation protocol was based partly on Cobb and Domain (2000). For the results presented in Figures 1 and 6, the following protocol was used. Half of the larvae collected from a food bottle were placed in a $35-\mathrm{mm}$ Petri dish in $600 \mu \mathrm{L}$ of Ringer's solution, and a filter paper square $(20 \mathrm{~mm}$ by $15 \mathrm{~mm}$ ) with $100 \mu \mathrm{L}$ of the selected odor was placed on the center of the lid. Larvae were left in the dish for $5 \mathrm{~min}$, and were thus preexposed to the odor prior to olfactory response testing. As a control, the other half of the larvae collected from the same food bottle underwent the same procedure, but with distilled water instead of an odor. For the results shown in Figures 2, 4, and 5, larvae were washed off multiple bottles and placed on two 500-mL flasks coated with $1 \%$ agar. Air was bubbled through the appropriate concentration of either diluted odorant or water for $5 \mathrm{~min}$. The two different procedures did not give us any significant differences in RI values and, where tested (Supplemental Table 1), for example, for GH146>Rdli, showed easily comparable and consistent results.

For exposure at $34^{\circ} \mathrm{C}$, odorant solution and the flask in which the larvae is introduced were pre-warmed in a water bath held at $34^{\circ} \mathrm{C}$. The exposed larvae were washed with lukewarm water and put in Ringer's solution. After 5 min of exposure, larvae were gently rinsed with lukewarm water, dried on filter paper, and placed in a fresh $35-\mathrm{mm}$ Petri dish in $600 \mu \mathrm{L}$ of Ringer's solution until the olfactory response tests (these took place within a few minutes, except in the case of spontaneous recovery experiments). In all cases, the larvae were tested as described in the previous section either at room temperature or in a warm room held at $34^{\circ} \mathrm{C}$.

\section{Stimulation with blue light for larval strains ectopically expressing channelrhodopsin}

The fly strains expressing channelrhodopsin were grown at $25^{\circ} \mathrm{C}$ on retinal medium and in the dark (as retinal is light-sensitive). Four microliters of $100 \mathrm{mM}$ retinal was added per milliliter of medium.

The light-gated cation channel, channelrhodopsin (ChR2), is most sensitive at $480 \mathrm{~nm}$, and, hence, neurons ectopically expressing ChR2 can be activated by blue-light exposure. Larvae were washed off the food bottles and places in a dish in Drosophila Ringer's solution. They were pulsed (15 sec on and 5 sec off) using a $5 \mathrm{~W}$ LED that emits at $480 \mathrm{~nm}$ (Luxeon-V Star Lumileds Lambertian Emitters) for $10 \mathrm{~min}$. Following photostimulation, the larvae were allowed to recover for $5 \mathrm{~min}$ and then tested for their olfactory response by the larval plate test, which is then compared to the naive response of the larvae to the same odorant to calculate the percent response (as in the previous sections). In control experiments, the larvae were similarly pulsed with red light ( $626 \mathrm{~nm}$; Luxeon LEDs), to which ChR2 is relatively insensitive, and their behavior was analyzed. As ChR2 is lightsensitive, larvae were grown and harvested in the dark.

\section{Thermoactivation of TrpAl-expressing neurons}

TrpA1 is a heat-sensitive cation channel that is closed at temperatures below $25^{\circ} \mathrm{C}$ and activated strongly at temperatures above 
$29^{\circ} \mathrm{C}$ (Rosenweig et al. 2005; Pulver et al. 2009). To induce STH in Or $83 b>\operatorname{TrpA} 1$ larvae, they were shifted from $21^{\circ} \mathrm{C}$ to $30^{\circ} \mathrm{C}$ for 30 min (as 10-min exposure did not cause a significant behavioral decrement). Control larvae were treated identically.

\section{Cold shock}

Following 5 min of odor exposure, larvae were rinsed and rested for $1 \mathrm{~min}$ in $1.5 \mathrm{~mL}$ of $4^{\circ} \mathrm{C}$ distilled water before being dried on filter paper, and collected into fresh Petri plates with Ringer's solution (room temperature) for olfactory response tests. Larvae exposed to water rather than odor underwent the same procedure as a control, as well as resting in $1.5 \mathrm{~mL}$ of water at room temperature post-exposure rather than $4^{\circ} \mathrm{C}$ water.

\section{MARCM experiments}

In order to generate single-cell MARCM clones of LNs, males of GAL4-LN2, UASmCD8::GFP/Y; FRTG13, UASmCD8::GFP/CyO were crossed to virgins of $\mathrm{y}$,w,hs-flp; FRTG13, tub-GAL80/Cyo flies. To generate the single PN clone, males of UAS-LacZ, UAS-mCD8::GFP/CyO-GFP; FRT82B were crossed to virgins of $y$, $w$,hs-flp; GAL4-GH146/CyO-GFP; FRT82B, tub-GAL80. Embryos from the above crosses were collected at 2-h intervals and reared at $25^{\circ} \mathrm{C}$. Heat shocks were given to the embryos at $3-5 \mathrm{~h}$ after egg laying (AEL) for $1 \mathrm{~h}$ in a water bath maintained at $37^{\circ} \mathrm{C}$. Cultures were returned to $25^{\circ} \mathrm{C}$, and animals were allowed to develop to third-instar larval stage when their brains were dissected and immunolabeled.

\section{Immunohistochemistry}

Larval brains were dissected in ice-cold $1 \times$ PBS and fixed in $4 \%$ PFA (prepared in $1 \times \mathrm{PBS}$ ) for $60 \mathrm{~min}$ at $4^{\circ} \mathrm{C}$. The fixative was then removed, and the brain samples were washed and blocked four times with $0.1 \%$ PBTX $(0.1 \%$ Triton X-100 and $0.1 \%$ BSA containing $1 \times$ PBS) for $15 \mathrm{~min}$ each at room temperature. Primary antibody (diluted in $0.1 \%$ PBTX) incubation was done for two overnights in $4^{\circ} \mathrm{C}$. The primary antibodies used were rabbit anti-GFP (1:10,000; Molecular Probes, Invitrogen), chicken antiGFP (1:500; AbCam), mouse anti-Bruchpilot (mAbnc82, 1:20; DSHB), rabbit anti-GABA (1:500; cat\#A2052, Sigma). After primary incubation, brains were washed in $0.1 \%$ PTX $(0.1 \%$ Triton X-100 containing PBS) for four times of 15 min each. Secondary antibody (diluted in $0.1 \%$ PTX) incubation was done for overnight in $4^{\circ} \mathrm{C}$, followed by similar wash with $0.1 \%$ PTX, followed by mounting in $70 \%$ glycerol. The secondary antibodies used were Alexa- 488 , Alexa-568, and Alexa-647 coupled antibodies generated in goat (Molecular Probes); all were used at 1:400 dilutions. Brain samples were imaged on an Olympus Fluoview FV1000 Laser Scanning Confocal microscope at $1-\mu \mathrm{m}$ intervals with a size of $512 \times 512$ pixels; data were processed using Olympus Fluoview (version 2.0), ImageJ, and Adobe Photoshop CS3 software.

\section{Acknowledgments}

This paper is dedicated to the memory of Veronica Rodrigues. She was a terrific scientist, superb mentor, and wonderful friend. We thank Andre Fiala, Erich Buchner, Martin Heisenberg, Ron Davis, Sean Sweeney, Li Liu, Tim Tully, Ann-Shyn Chiang, and Leslie Vosshall for Drosophila stocks and reagents, and K.S. Krishnan and Tomas Ryan for early help with larval behavior. We thank Eimear Holohan, David Glanzman, Adrian Dervan, and K. VijayRaghavan for useful discussions and comments on the manuscript, and Sudeshna Das for useful discussions as well as help in plotting some of the graphs in the figures. This work was funded by grants from the Science Foundation of Ireland (to M.R.) and the Indian Department of Biotechnology (to V.R.). We acknowledge seed support from an NIDA FIRCA Supplement Award to M.R. and core funding from the DAE to the TIFR and NCBS.

\section{References}

Aceves-Pina EO, Quinn WG. 1979. Learning in normal and mutant Drosophila larvae. Science 206: 93-96.
Armitage BA, Siegelbaum SA. 1998. Presynaptic induction and expression of homosynaptic depression at Aplysia sensorimotor neuron synapses. J Neurosci 18: 8770-8779.

Asztalos Z, Arora N, Tully T. 2007. Olfactory jump reflex habituation in Drosophila and effects of classical conditioning mutations. J Neurogenet 21: $1-18$.

Bailey CH, Chen M. 1983. Morphological basis of long-term habituation and sensitization in Aplysia. Science 220: 91-93.

Bailey CH, Chen M. 1988. Morphological basis of short-term habituation in Aplysia. J Neurosci 8: 2452-2459.

Blum AL, Li W, Cressy M, Dubnau J. 2009. Short- and long-term memory in Drosophila require cAMP signaling in distinct neuron types. Curr Biol 19: $1341-1350$.

Bourne HR, Nicoll R. 1993. Molecular machines integrate coincident synaptic signals. Cell 72: $65-75$.

Boyle J, Cobb M. 2005. Olfactory coding in Drosophila larvae investigated by cross-adaptation. J Exp Biol 208: 3483-3491.

Cho W, Heberlein U, Wolf FW. 2004. Habituation of an odorant-induced startle response in Drosophila. Genes Brain Behav 3: 127-137.

Chou YH, Spletter ML, Yaksi E, Leong JC, Wilson RI, Luo L. 2010. Diversity and wiring variability of olfactory local interneurons in the Drosophila antennal lobe. Nat Neurosci 13: 439-449.

Cobb M, Domain I. 2000. Olfactory coding in a simple system: Adaptation in Drosophila larvae. Proc Biol Sci 267: 2119-2125.

Duerr JS, Quinn WG. 1982. Three Drosophila mutations that block associative learning also affect habituation and sensitization. Proc Natl Acad Sci 79: 3646-3650.

Engel JE, Wu CF. 1996. Altered habituation of an identified escape circuit in Drosophila memory mutants. J Neurosci 16: 3486-3499.

Engel JE, Wu CF. 2009. Neurogenetic approaches to habituation and dishabituation in Drosophila. Neurobiol Learn Mem 92: 166-175.

Fabiani M, Low KA, Wee E, Sable JJ, Gratton G. 2006. Reduced suppression or labile memory? Mechanisms of inefficient filtering of irrelevant information in older adults. J Cogn Neurosci 18: 637-650.

Fishilevich E, Domingos AI, Asahina K, Naef F, Vosshall LB, Louis M. 2005. Chemotaxis behavior mediated by single larval olfactory neurons in Drosophila. Curr Biol 15: 2086-2096.

Frankland PW, Wang Y, Rosner B, Shimizu T, Balleine BW, Dykens EM, Ornitz EM, Silva AJ. 2004. Sensorimotor gating abnormalities in young males with fragile X syndrome and Fmr1-knockout mice. Mol Psychiatry 9: 417-425.

Freedman R, Waldo M, Bickford-Wimer P, Nagamoto H. 1991. Elementary neuronal dysfunctions in schizophrenia. Schizophr Res 4: 233-243.

Gervasi N, Tchenio P, Preat T. 2010. PKA dynamics in a Drosophila learning center: Coincidence detection by Rutabaga adenylyl cyclase and spatial regulation by Dunce phosphodiesterase. Neuron 65: 516-529.

Glanzman DL. 2009. Habituation in Aplysia: The Cheshire cat of neurobiology. Neurobiol Learn Mem 92: 147-154.

Glanzman DL, Epperlein RC. 1981. Disruption of vertebrate monosynaptic habituation by ethyl alcohol. Brain Res 212: 117-126.

Groves PM, Thompson RF. 1970. Habituation: A dual-process theory. Psychol Rev 77: 419-450.

Hildebrand JG, Shepherd GM. 1997. Mechanisms of olfactory discrimination: Converging evidence for common principles across phyla. Annu Rev Neurosci 20: 595-631.

Hunt PS, Morasch KC. 2004. Modality-specific impairments in response habituation following postnatal binge ethanol. Neurotoxicol Teratol 26: $451-459$.

Kitamoto T. 2001. Conditional modification of behavior in Drosophila by targeted expression of a temperature-sensitive shibire allele in defined neurons. J Neurobiol 47: 81-92.

Kitamoto T. 2002. Targeted expression of temperature-sensitive dynamin to study neural mechanisms of complex behavior in Drosophila. J Neurogenet 16: 205-228.

Krasne FB, Teshiba TM. 1995. Habituation of an invertebrate escape reflex due to modulation by higher centers rather than local events. Proc Natl Acad Sci 92: 3362-3366.

Kullmann DM, Lamsa KP. 2007. Long-term synaptic plasticity in hippocampal interneurons. Nat Rev Neurosci 8: 687-699.

Larsson MC, Domingos AI, Jones WD, Chiappe ME, Amrein H, Vosshall LB. 2004. Or83b encodes a broadly expressed odorant receptor essential for Drosophila olfaction. Neuron 43: 703-714.

Levin LR, Han PL, Hwang PM, Feinstein PG, Davis RL, Reed RR. 1992. The Drosophila learning and memory gene rutabaga encodes a $\mathrm{Ca}^{2+} /$ calmodulin-responsive adenylyl cyclase. Cell 68: 479-489.

Linster C, Henry L, Kadohisa M, Wilson DA. 2007. Synaptic adaptation and odor-background segmentation. Neurobiol Learn Mem 87: $352-360$.

Lisman JE, Coyle JT, Green RW, Javitt DC, Benes FM, Heckers S, Grace AA. 2008. Circuit-based framework for understanding neurotransmitter and risk gene interactions in schizophrenia. Trends Neurosci 31: 234-242. 
Liu X, Krause WC, Davis RL. 2007. GABAA receptor RDL inhibits Drosophila olfactory associative learning. Neuron 56: 1090-1102.

Liu X, Buchanan ME, Han KA, Davis RL. 2009. The GABAA receptor RDL suppresses the conditioned stimulus pathway for olfactory learning. $J$ Neurosci 29: 1573-1579.

Livingstone MS, Sziber PP, Quinn WG. 1984. Loss of calcium/calmodulin responsiveness in adenylate cyclase of rutabaga, a Drosophila learning mutant. Cell 37: 205-215.

Ludewig K, Geyer MA, Vollenweider FX. 2003. Deficits in prepulse inhibition and habituation in never-medicated, first-episode schizophrenia. Biol Psychiatry 54: 121-128.

Malenka RC, Bear MF. 2004. LTP and LTD: An embarrassment of riches. Neuron 44: 5-21.

Masse NY, Turner GC, Jefferis GS. 2009. Olfactory information processing in Drosophila. Curr Biol 19: R700-R713.

McNamara AM, Magidson PD, Linster C, Wilson DA, Cleland TA. 2008. Distinct neural mechanisms mediate olfactory memory formation at different timescales. Learn Mem 15: 117-125.

Meincke U, Light GA, Geyer MA, Braff DL, Gouzoulis-Mayfrank E. 2004. Sensitization and habituation of the acoustic startle reflex in patients with schizophrenia. Psychiatry Res 126: 51-61.

Monte P, Woodard C, Ayer R, Lilly M, Sun H, Carlson J. 1989. Characterization of the larval olfactory response in Drosophila and its genetic basis. Behav Genet 19: 267-283.

Oda Y, Kawasaki K, Morita M, Korn H, Matsui H. 1998. Inhibitory long-term potentiation underlies auditory conditioning of goldfish escape behaviour. Nature 394: $182-185$.

Okada R, Awasaki T, Ito K. 2009. Gamma-aminobuyric acid (GABA)-mediated neural connections in the Drosophila antennal lobe. J Comp Neurol 514: 74-91.

Olsen SR, Wilson RI. 2008. Cracking neural circuits in a tiny brain: New approaches for understanding the neural circuitry of Drosophila. Trends Neurosci 31: 512-520.

Ornitz EM, Lane SJ, Sugiyama T, de Traversay J. 1993. Startle modulation studies in autism. I Autism Dev Disord 23: 619-637.

Pulver SR, Pashkovski SL, Hornstein NJ, Garrity PA, Griffith LC. 2009. Temporal dynamics of neuronal activation by Channelrhodopsin-2 and TRPA1 determine behavioral output in Drosophila larvae. $J$ Neurophysiol 101: 3075-3088.

Ramaekers A, Magnenat E, Marin EC, Gendre N, Jefferis GS, Luo L, Stocker RF. 2005. Glomerular maps without cellular redundancy at successive levels of the Drosophila larval olfactory circuit. Curr Biol 15: 982-992.

Rankin CH, Abrams T, Barry RJ, Bhatnagar S, Clayton DF, Colombo J, Coppola G, Geyer MA, Glanzman DL, Marsland S, et al. 2009. Habituation revisited: An updated and revised description of the behavioral characteristics of habituation. Neurobiol Learn Mem 92: $135-138$.

Rodrigues V. 1980. Olfactory behavior of Drosophila melanogaster. In Development and neurobiology of Drosophila (ed. O Siddiqi et al.), pp. 361-371. Plenum, New York.
Rosenzweig M, Brennan KM, Tayler TD, Phelps PO, Patapoutian A, Garrity PA. 2005. The Drosophila ortholog of vertebrate TRPA1 regulates thermotaxis. Genes Dev 19: 419-424.

Sachse S, Rueckert E, Keller A, Okada R, Tanaka NK, Ito K, Vosshall LB. 2007. Activity-dependent plasticity in an olfactory circuit. Neuron 56: $838-850$.

Schroll C, Riemensperger T, Bucher D, Ehmer J, Voller T, Erbguth K, Gerber B, Hendel T, Nagel G, Buchner E, et al. 2006. Light-induced activation of distinct modulatory neurons triggers appetitive or aversive learning in Drosophila larvae. Curr Biol 16: 1741-1747.

Siniatchkin M, Kropp P, Gerber WD. 2003. What kind of habituation is impaired in migraine patients? Cephalalgia 23: 511-518.

Slaats-Willemse D, Swaab-Barneveld H, de Sonneville L, van der Meulen E, Buitelaar J. 2003. Deficient response inhibition as a cognitive endophenotype of ADHD. J Am Acad Child Adolesc Psychiatry 42: $1242-1248$

Stocker RF. 2008. Design of the larval chemosensory system. Adv Exp Med Biol 628: 69-81.

Suh GS, Ben-Tabou de Leon S, Tanimoto H, Fiala A, Benzer S, Anderson DJ. 2007. Light activation of an innate olfactory avoidance response in Drosophila. Curr Biol 17: 905-908.

Sweeney ST, Broadie K, Keane J, Niemann H, O'Kane CJ. 1995. Targeted expression of tetanus toxin light chain in Drosophila specifically eliminates synaptic transmission and causes behavioral defects. Neuron 14: $341-351$.

Tanaka NK, Ito K, Stopfer M. 2009. Odor-evoked neural oscillations in Drosophila are mediated by widely branching interneurons. J Neurosci 29: $8595-8603$.

Thompson RF, Spencer WA. 1966. Habituation: A model phenomenon for the study of neuronal substrates of behavior. Psychol Rev 73: 16-43.

Vosshall LB, Stocker RF. 2007. Molecular architecture of smell and taste in Drosophila. Annu Rev Neurosci 30: 505-533.

Wilson DA, Linster C. 2008. Neurobiology of a simple memory. J Neurophysiol 100: 2-7.

Wolf FW, Eddison M, Lee S, Cho W, Heberlein U. 2007. GSK-3/Shaggy regulates olfactory habituation in Drosophila. Proc Natl Acad Sci 104: $4653-4657$.

Wu CL, Xia S, Fu TF, Wang H, Chen YH, Leong D, Chiang AS, Tully T. 2007. Specific requirement of NMDA receptors for long-term memory consolidation in Drosophila ellipsoid body. Nat Neurosci 10: $1578-1586$.

Wuttke MS, Tompkins L. 2000. Olfactory adaptation in Drosophila larvae. J Neurogenet 14: 43-62.

Xia S, Miyashita T, Fu TF, Lin WY, Wu CL, Pyzocha L, Lin IR, Saitoe M, Tully T, Chiang AS. 2005. NMDA receptors mediate olfactory learning and memory in Drosophila. Curr Biol 15: 603-615.

Zars T, Fischer M, Schulz R, Heisenberg M. 2000. Localization of a short-term memory in Drosophila. Science 288: 672-675.

Received April 20, 2010; accepted in revised form September 21, 2010. 


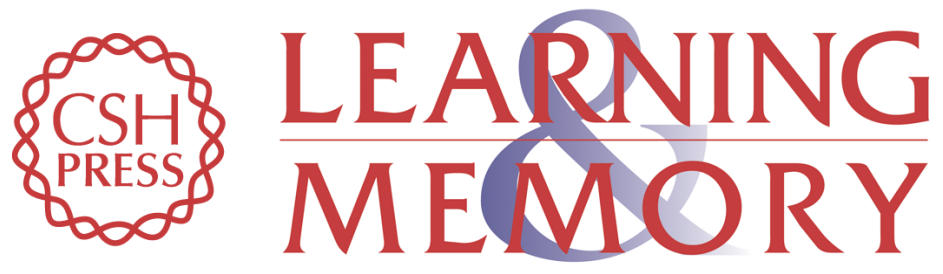

\section{Central synaptic mechanisms underlie short-term olfactory habituation in Drosophila larvae}

Aoife Larkin, Somdatta Karak, Rashi Priya, et al.

Learn. Mem. 2010, 17:

Access the most recent version at doi:10.1101//m.1839010

Supplemental http://learnmem.cshlp.org/content/suppl/2010/11/24/17.12.645.DC1
Material

References This article cites 65 articles, 14 of which can be accessed free at: http://learnmem.cshlp.org/content/17/12/645.full.html\#ref-list-1

License

Email Alerting

Receive free email alerts when new articles cite this article - sign up in the box at the Service top right corner of the article or click here. 